\title{
Temporal integration of acoustic and cutaneous stimuli shown in the blink reflex
}

\author{
YVONNE PLANT and GEOFFREY R. HAMMOND \\ The University of Western Australia, Nedlands, Western Australia, Australia
}

\begin{abstract}
Temporal integration of pairs of brief blink-eliciting acoustic and cutaneous stimuli was investigated to determine if there was integration of stimuli from different modalities. Reflexes elicited by a tone burst or by a brief electrical shock to the supraorbital nerve followed by a second tone burst or shock at short stimulus onset asynchronies (SOAs) were larger and faster than control reflexes elicited by a single stimulus identical to the lead stimulus of the stimulus pairs. Reflex amplitude was augmented at longer SOAs where there was no effect on latency. Temporal integration was evident for all stimulus pairs, showing that it is due, at least in part, to processes that occur outside specific sensory pathways. Heterogeneous stimulus pairs produced greater reflex enhancement than did homogeneous stimulus pairs. This finding was examined further in Experiment 2, which showed that reflex enhancement with pairs of acoustic pulses was unaffected by the frequency of the second stimulus, suggesting that sensory masking was not acting to suppress reflex expression with acoustic pulse pairs. Integration of reflexogenic acoustic stimuli shown in the blink reflex is restricted to shorter intervals than is integration of acoustic stimuli shown by psychophysical procedures, suggesting that the two methods reflect different aspects of stimulus processing. Integration of reflexogenic stimuli may result from summation of activity associated more directly with reflex expression than with perceptual awareness.
\end{abstract}

The blink component of the acoustic startle reflex in humans has been used to study integration of stimulation distributed in time. K. M. Berg (1973) found that acoustic startle blink thresholds decreased as the duration of the eliciting tone burst increased from 4 to $32 \mathrm{msec}$. More recently, Blumenthal and W. K. Berg (1986) reported similar findings with blink reflex amplitude, using both continuous acoustic stimuli of varying durations and acoustic pulse pairs with varying stimulus onset asynchronies (SOAs). They showed that reflex amplitude increased as the duration of a continuous noise burst increased from 3 to about $50 \mathrm{msec}$ and was asymptotic with further increases in stimulus duration to $100 \mathrm{msec}$. Reflex latency decreased as stimulus duration increased from 3 to $20 \mathrm{msec}$. Pairs of 3-msec noise bursts with SOAs from 20 to 40 msec produced greater reflex amplitudes than did single 3-msec stimuli. At SOAs longer than $50 \mathrm{msec}$, reflex amplitudes in the paired pulse condition declined to the control level elicited by the single 3-msec pulse. There was no systematic effect of SOA (from 20 to $100 \mathrm{msec}$ ) on reflex latency.

These results show that both prolonging stimulus duration and presenting closely spaced pulse pairs increase reflex amplitude, that reflex amplitude becomes asymptotic beyond a certain continuous stimulus duration, and that reflex amplitude declines beyond a certain pulse-pair SOA. Blumenthal and W. K. Berg (1986) argued that the increased reflex amplitude resulted from integration by the auditory system of stimulation within a critical time

Send reprint requests to Geoffrey R. Hammond, Department of Psychology, The University of Western Australia, Nedlands, Western Australia 6009, Australia. period. They also argued that the continuous stimuli and the pulse pairs were processed by separate functional systems within the auditory pathway specialized for processing sustained and transient stimulus characteristics, and that the transient-processing system was more efficient because reflex amplitudes elicited by the brief $(3-\mathrm{msec})$ pulse pairs with SOAs from 20 to $40 \mathrm{msec}$ were similar to those elicited by the corresponding continuous stimuli with durations from 20 to $40 \mathrm{msec}$.

These transient and sustained systems were identified with neurons such as the short-time-constant and long-time-constant neurons described in the cat auditory system by Gersuni (1971) and Radionova (1971). Long-time-constant neurons showed tonic firing throughout the duration of an auditory signal, and short-timeconstant neurons showed phasic bursts of firing at the onset and offset of a signal. These neuron types, however, may not form specialized systems responsible for the integration of continuous and pulse-pair stimuli shown by Blumenthal and W. K. Berg (1986). The differences in response characteristics of these neuron types are most apparent at threshold stimulation levels and are not necessarily maintained at high intensity levels (Radionova, 1971). Radionova also noted that phasic neurons usually displayed tonic discharge patterns at suprathreshold intensity levels, which suggests that the two neuronal types respond differently only to near-threshold stimuli and not to stimuli as intense as those used to evoke startle.

\section{EXPERIMENT 1}

Experiment 1 was conducted to determine whether temporal integration of pulse pairs shown in the blink reflex 
is restricted to auditory stimuli or occurs more generally. The blink reflex can be elicited readily by cutaneous stimulation, including mechanical stimulation of the periorbital region and electrical stimulation of the supraorbital branch of the trigeminal nerve. The electrically elicited cutaneous blink reflex is characterized by an early brief electromyographic (EMG) component ipsilateral to the eliciting stimulus and a later, more prolonged, bilateral EMG component (Penders \& Delwaide, 1973; Shahani \& Young, 1973). The second component is associated with eyelid closure and is considered analogous to the single component of the acoustic blink reflex (Shahani \& Young, 1973).

Subjects were presented with stimulus pairs composed of acoustic (A) and cutaneous (C) reflex-eliciting stimuli. If temporal integration were restricted to specific sensory auditory neurons, it would be restricted to A-A pairs; if temporal integration were due to a sensory process that operates in both auditory and cutaneous systems, it would be evident with the A-A and the C-C pairs, but not with the A-C or the C-A pairs; and if temporal integration were not restricted to processes within specific sensory pathways, it would be evident in all stimulus conditions.

\section{Method}

Subjects. Eight student volunteers were subjects. The 4 males and 4 females, aged from 18 to 32 years (median $=21$ ), had no prior knowledge of the experiment and no history of hearing or neurological disorders or of headaches.

Apparatus and Procedure. The subject was seated in a comfortable reclining chair in a sound-attenuated room. Miniature stainless steel stimulating electrodes were attached to the skin over the right supraorbital nerve. One electrode was placed directly above the eyebrow in a line with the supraorbital notch, and the other was placed about $1.5 \mathrm{~cm}$ above the first. EMG activity was recorded with gold-plated electrodes from the left orbicularis oculi muscle. The two recording electrodes were placed at the lateral canthus and just below the lower lid margin in line with the pupil when it looked straight ahead. A ground electrode was placed midway between the two recording electrodes on the zygomatic arch. Recordings were taken from the side contralateral to stimulation to restrict measurements to the second EMG component of the cutaneous reflex. This procedure also reduced stimulus artifact in the recordings. The impedance between the ground and recording electrodes was between 5 and $12 \mathrm{k} \Omega$ for all subjects.

Individual reflex thresholds for the acoustic and the cutaneous stimuli were then determined. Acoustic stimuli were 3-msec 0.9-kHz tone bursts produced by a Wavetek function generator that was gated on by a square wave from a Grass SD9 stimulator under microcomputer control. The output of the function generator was enabled at the first zero crossing of the sine wave following gate onset, and disabled at the first zero crossing of the sine wave following gate offset. The stimuli were presented binaurally through headphones. Single stimuli were presented at 20 - to 40 -sec intervals, beginning at $70 \mathrm{dBA}$ and increasing in about 2-dB steps until three consecutive EMG responses were obtained at one intensity. Stimulus intensities for the different subjects ranged from 72 to $93 \mathrm{dBA}$, with a mean of $84 \mathrm{dBA}$. Threshold intensity for electrocutaneous stimulation of the supraorbital nerve was then determined in a similar way. Cutaneous stimuli were 0.5 -msec biphasic square-wave shocks produced by a Grass SD9 stimulator and routed through a Grass constant-current unit. Single stimuli were presented to the supra- orbital nerve at intertrial intervals of 20-40 sec, beginning at $0.5 \mathrm{~mA}$ and increasing in about $0.2-\mathrm{mA}$ steps until a blink was elicited on three consecutive presentations. Threshold intensities for the individual subjects ranged from 1.5 to $5.2 \mathrm{~mA}$, with a mean of $3.6 \mathrm{~mA}$. These individual acoustic and cutaneous stimulus intensities were maintained throughout the experiment.

The subjects were given reading materials to promote stable attention and to retard habituation. The subjects were asked to sit quietly during the experiment and to move, when necessary, within the few seconds after a stimulus presentation. Reflex-eliciting stimulus pairs were presented in four combinations: acoustic-acoustic (A-A), acoustic-cutaneous (A-C), cutaneous-cutaneous (C-C), and cutaneous-acoustic (C-A). Within each stimulus-pair condition, the pulse pairs were presented at SOAs of $3,9,18,36$, and $72 \mathrm{msec}$. Single auditory and cutaneous stimuli identical to the leading stimuli of the stimulus pairs were also given to establish control reflex values. Therefore, there were 24 possible stimulus types (four stimulus-pair conditions, each with five paired stimuli and an appropriate single stimulus). The presentation sequence of the stimulus types was determined by a $24 \times 24$ incomplete counterbalanced square design (D'Amato, 1979) in which all 24 stimulus types were represented in each row. This design ensured that each stimulus type was presented only once at each position in the row and was never preceded by the same stimulus type more than once. Each subject completed six rows (six trials at each of the 24 stimulus types) for a total of 144 trials. The starting rows for the first and fourth subjects were decided randomly, and, once those were determined, the starting rows for the remaining subjects were predetermined.

The intertrial intervals varied randomly from 20 to $40 \mathrm{sec}$, with a mean of $30 \mathrm{sec}$. The 72-min, 144-trial session was divided into three 24-min blocks of 48 trials each to minimize fatigue and habituation. An 8-min rest period was given between each block.

Data recording and analysis. The EMG signals were amplified by a Grass 7P511 amplifier and sent to an 8-bit analog-to-digital converter of a microcomputer, where they were sampled and digitized every millisecond. Digitized amplitude scores were saved during a time window of $25-105 \mathrm{msec}$ after the onset of the first stimulus in a pair to limit the scoring to stimulus-elicited blinks. For each of the six trials, the peak-to-peak reflex amplitudes (in millivolts) produced by the paired stimuli in each condition were expressed as a ratio of the control reflex amplitude elicited by the single stimulus in that trial block. This was done separately for each of the six trials in each of the four conditions. These amplitude ratios were transformed logarithmically for analyses of variance, and geometric means of the ratios are reported. Reflex latency was scored directly from a storage oscilloscope display as the time in milliseconds from the onset of the first stimulus in each pair to the onset of the first negative peak of the EMG response. Trials contaminated with EMG activity in the 20 msec before stimulus onset were discarded. These accounted for $2.5 \%$ of all trials.

\section{Results}

Reflex amplitude. The mean proportional peak-to-peak amplitudes from the four stimulus-pair conditions at the five SOAs are shown in Figure 1. The mean reflex amplitudes produced by paired stimuli were greater than those elicited by single stimuli at each SOA in all four conditions. This augmentation of reflex amplitude with paired stimuli was greatest at the shorter SOAs $(3,9$, and $18 \mathrm{msec}$ ) and least at the longest SOA $(72 \mathrm{msec})$. The time course of reflex augmentation was similar for the different stimulus combinations. Reflex amplitudes decreased as the SOA increased beyond $18 \mathrm{msec}$ and were only 


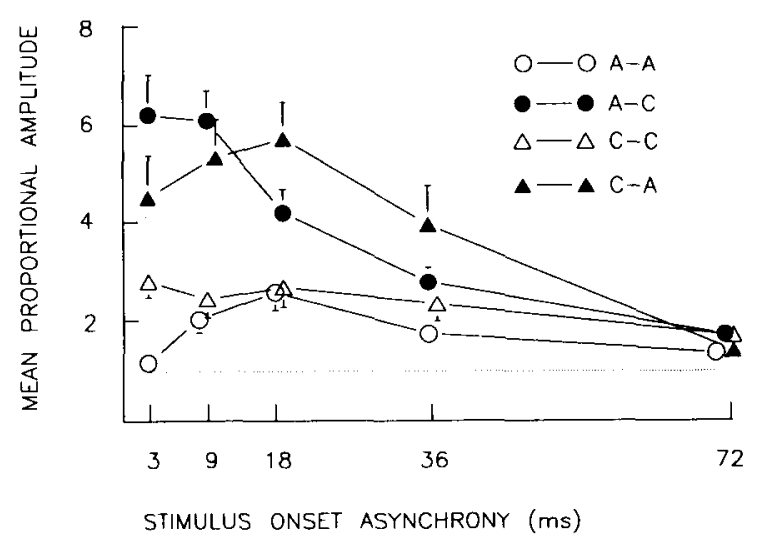

Figure 1. Geometric means of the proportional reflex amplitudes at each of the five stimulus onset asynchronies in Experiment 1. The dotted line shows a proportional amplitude of 1.0 . The vertical bars show one standard error of the mean where it is larger than symbol size.

slightly greater than single-stimulus amplitudes at the 72-msec SOA. Increased reflex amplitude was not present at the 3-msec SOA in the A-A condition, but emerged at longer SOAs and was greatest at the 18 -msec SOA. The main effect of SOA was statistically significant $[F(4,28)=59.70, p<.05]$. One-tailed Dunnett's $t$ tests were used to compare the mean reflex amplitudes of all the stimulus pairs with the mean control reflex amplitudes of the single stimuli. The same patterns of significance were found for the A-C, C-C, and C-A conditions: mean reflex amplitudes elicited in these conditions at SOAs from 3 to $36 \mathrm{msec}$ inclusive were significantly greater than the control amplitude elicited by the single stimulus. The amplitudes elicited at the 72-msec SOA were not significantly greater than the control amplitude. The A-A condition showed a different pattern of significance. The reflex amplitudes produced by pulse pairs with 9 - and $18-\mathrm{msec}$ SOAs were significantly greater than single-stimulus amplitudes. Reflex amplitude was not significantly greater than the single-stimulus amplitude at all other SOAs.

Amplitude augmentation was greater in the heterogeneous stimulus-pair conditions (A-C and $\mathrm{C}-\mathrm{A}$ ) than in the homogeneous stimulus-pair conditions (A-A and $\mathrm{C}$ C). Maximum amplitude augmentation was about six times the single-stimulus levels for the heterogeneous stimulus pairs and about three times the single-stimulus levels for the homogeneous stimulus pairs. The main effect of stimulus-pair homogeneity was statistically significant $[F(1,7)=18.92, p<.05]$. There was also a significant stimulus-pair homogeneity $\times$ SOA interaction $[F(4,28)=27.85, p<.05]$. The effect of stimulus-pair homogeneity was greatest at the shortest SOAs (where amplitude augmentation was greatest) and absent at the longest SOA (where there was no augmentation of amplitude).

Reflex latency. The mean reflex latencies from the four stimulus conditions at the five SOAs and for the singlestimulus presentations are shown in Figure 2. Latency was generally shorter than single-stimulus values at the brief SOAs. Latencies were shortest for the A-A pairs (except at the 3-msec SOA), intermediate for the A-C and C-A pairs, and longest for the $\mathrm{C}-\mathrm{C}$ pairs. There was a significant main effect of SOA $[F(4,28)=13.02, p<.05]$. One-tailed Dunnett's $t$ tests were used again to compare the mean latencies of the reflexes produced by the stimulus pairs with the single-stimulus control latencies. In the $\mathrm{A}-\mathrm{C}$ and $\mathrm{C}-\mathrm{A}$ conditions, the mean latencies at the $3-$ and 9-msec SOAs were significantly shorter than the corresponding control latencies; in the $\mathrm{C}-\mathrm{C}$ condition, the mean latency at the 3-msec SOA was significantly shorter than control; and in the A-A condition, the mean latencies at the 9- and 18-msec SOAs were significantly shorter than control. The upper limits for temporal integration shown by reflex latency are shorter than those described above for reflex amplitude.

\section{Discussion}

The results of Experiment 1 show an SOA-dependent reflex enhancement. Reflexes elicited by stimulus pairs with short SOAs were larger and faster than reflexes elicited by the corresponding single stimulus. The results with the paired acoustic stimuli replicate those of Blumenthal and W. K. Berg (1986) and are consistent with previous reports of startle amplitude augmentation with two closely spaced acoustic stimuli in rats (Marsh, Hoffman, \& Stitt, 1973). Augmentation of reflex amplitude by a second acoustic stimulus that follows the eliciting stimulus closely in time has been attributed to temporal integration of the two stimuli (Blumenthal \& W. K. Berg, 1986; Marsh et al., 1973). Reflex enhancement was also evident in the present experiments when an electrical pulse to the supraorbital nerve (the cutaneous stimulus) was followed closely by another cutaneous stimulus, showing that

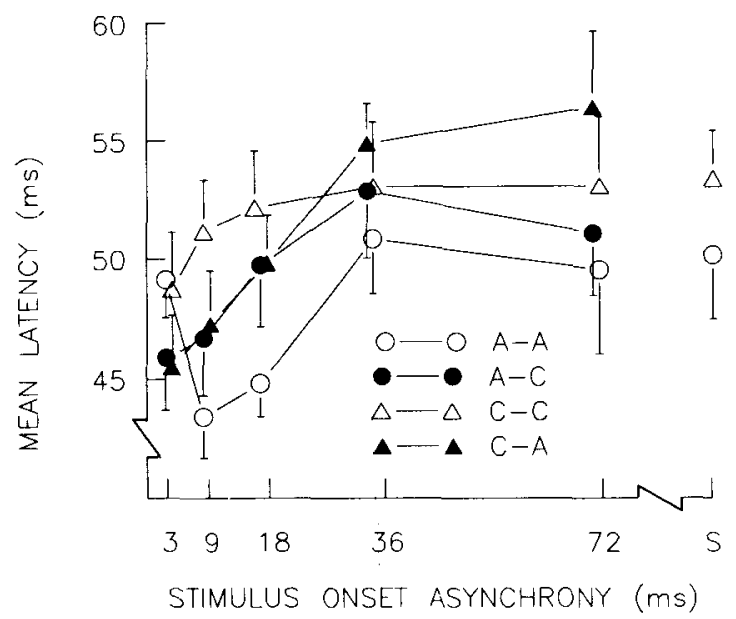

Figure 2. Mean reflex latencies (measured in milliseconds from the first stimulus) at each of the five stimulus onset asynchronies in Experiment 1. Single-stimulus latencies (S) for the acoustic stimuli (open circle) and cutaneous stimuli (open triangle) are shown on the right of the figure. The vertical bars show one standard error of the mean. 
temporal integration is not restricted to the auditory modality. The most important finding is that there was temporal integration of two stimuli from different sensory modalities. An acoustic stimulus followed quickly by a cutaneous stimulus elicited a larger and faster reflex than a single acoustic stimulus, and a cutaneous stimulus followed quickly by an acoustic stimulus elicited a larger and faster reflex than a single cutaneous stimulus. In both the $\mathrm{A}-\mathrm{C}$ and $\mathrm{C}-\mathrm{A}$ conditions, reflex enhancement was a systematic function of the SOA. These findings show clearly that temporal integration shown by reflex enhancement is not restricted to specific auditory neurons because it occurs in another sensory modality and, more importantly, it occurs between sensory modalities. Specific sensory integrative mechanisms do not give a complete account of the integration effects reported here, and, at least in part, the effects must be due to stimulus integration occurring outside specific sensory pathways. The decline of reflex amplitude to near control levels at the longest SOA is unlikely to be due to activation of the middle-ear reflex by the leading stimulus (hence attenuation of the impact of a trailing acoustic stimulus) as it was present with trailing cutaneous stimuli.

The present results support the suggestion of Blumenthal and W. K. Berg (1986) that latency facilitation with acoustic pulse pairs might be present at SOAs shorter than $20 \mathrm{msec}$. Although the average reflex latency at the 3-msec SOA in the A-A condition was not different from that of the single acoustic stimulus, latencies at the 9- and 18-msec SOAs were shorter than in the control condition. There was no effect on latency at the two longer SOAs. In the remaining conditions the latency of the reflex elicited by the first stimulus was shortened by a second stimulus presented at SOAs up to $9 \mathrm{msec}$ (A-C and C-A) or only at the 3-msec SOA (C-C). Temporal integration of pulse pairs is therefore evident in reflex latency, and occurs in the case in which each member of the pair is from a different sensory modality.

The absence of reflex enhancement at the shortest (3$\mathrm{msec}) \mathrm{SOA}$ in the A-A condition is similar to the absence of temporal integration in rats with 1-msec acoustic stimuli delivered with a 1-msec SOA (Marsh et al., 1973). Temporal integration was greatest in the present experiment at the 3-msec SOA in the A-C and C-A conditions (where the stimuli did not share common receptors) and in the C-C condition (where the receptors were bypassed by direct nerve stimulation), suggesting that the absence of reflex enhancement at the shortest SOA in the A-A condition was a result of reduced receptor excitability.

\section{EXPERIMENT 2}

The finding in Experiment 1 that homogeneous stimulus pairs resulted in less amplitude augmentation than did heterogeneous stimulus pairs might have resulted from masking between members of homogeneous stimulus pairs. Although the abrupt onset and offset of the brief tone bursts in Experiment 1 would have resulted in energy splatter that would have limited masking, it is still possible that some masking was present and attenuated the impact of either member of homogeneous acoustic and cutaneous stimulus pairs. In audition, masking between tones is frequency dependent and is greatest when the two tones are the same frequency (Green \& Wier, 1984). If masking does affect the reflexes elicited with paired acoustic stimuli, then separating their frequencies might release masking and enhance reflex expression. This experiment determined whether the blink reflex elicited by the first member of a closely spaced pair of tone bursts is a function of their respective frequencies.

\section{Method}

Subjects. Eight volunteers were selected from the same population with the same criteria used in Experiment 1 . There were 4 males and 4 females aged from 18 to 24 years (median $=21$ ).

Apparatus and Procedure. Two stimulus-pair conditions were tested: acoustic-acoustic (A-A) and cutaneous-acoustic (C-A). The procedures were similar to those followed in Experiment 1. The threshold intensities for individual subjects ranged from 73 to $85 \mathrm{dBA}$ with a mean of $81 \mathrm{dBA}$ for the acoustic stimuli, and from 2.0 to $5.3 \mathrm{~mA}$ with a mean of $2.9 \mathrm{~mA}$ for the cutaneous stimuli.

The stimuli were identical to those in Experiment 1 except for the frequency of the acoustic stimuli. In the A-A pairs the first stimulus was always a tone with a frequency of $4.0 \mathrm{kHz}$. The second stimulus in both stimulus-pair conditions was a tone with a frequency of $0.6,0.8,1.0,2.0$, or $4.0 \mathrm{kHz}$. The acoustic stimuli were generated by an Exact function generator with a voltage-controlled oscillator (VCO). Voltage levels established on the digital-to-analog converter of the microcomputer were routed to the VCO input of the function generator and determined the frequency of the output signal. A single stimulus equivalent to the first stimulus in the pair (A or C) was presented to establish control reflex levels for each condition. There were five paired stimuli and one single stimulus in each condition, making a total of 12 stimulus types (two stimulus pairs each with five $\mathbf{S} 2$ frequencies together with the two single stimuli). All stimulus pairs were presented with an SOA of $18 \mathrm{msec}$, which had been shown to produce reflex enhancement in Experiment 1 .

Trial sequence was determined by a $12 \times 12$ incomplete counterbalanced square design. Starting rows for each subject were decided randomly, and each subject completed six successive rows to give 6 trials at each of the 12 stimulus types. Each subject received a total of 72 trials. Intertrial intervals varied randomly from 20 to $40 \mathrm{sec}$ with a mean of $30 \mathrm{sec}$. The total 36 -min session was divided into two 18-min blocks of 36 trials each, with an 8-min rest period between to minimize fatigue and habituation.

When reflex measurements were completed, all subjects were given a brief psychophysical task to determine the relationship between $S 2$ frequency and loudness. This was done to establish whether any effect of $\$ 2$ frequency on reflex amplitude was secondary to changes in loudness. The subjects listened to pairs of tone bursts presented over headphones and rated the loudness of the second tone burst as a percentage of the loudness of the first. The frequency of the first stimulus was maintained at $4.0 \mathrm{kHz}$, and the frequency of the second stimulus was $0.6,0.8,1.0$, or $2.0 \mathrm{kHz}$. The tones were presented with an interstimulus interval of $500 \mathrm{msec}$ so that they were detected as two separate stimuli. Each of the four stimulus pairs was presented five times in a random order for a total of 
20 estimates. The intertrial interval was $8 \mathrm{sec}$, during which time the subjects wrote down their loudness estimates of the second stimulus.

\section{Results}

Reflex amplitude. The mean proportional peak-to-peak reflex amplitudes at the five $S 2$ frequencies in the two stimulus-pair conditions are shown in Figure 3. The amplitudes of the reflexes elicited by an acoustic or cutaneous stimulus followed by an acoustic S2 after an SOA of $18 \mathrm{msec}$ were greater than those elicited by a single acoustic or cutaneous stimulus. As in Experiment 1, this amplitude augmentation was more pronounced when the two stimuli were presented in different modalities (C-A) than in the same modality (A-A). Amplitude augmentation was about five times the control level for the $\mathrm{C}$-A pairs at all $S 2$ frequencies and about twice the control level for the A-A pairs at all S2 frequencies. The main effect of stimulus condition was significant $[F(1,7)=21.68, p<.05]$.

The frequency of the second stimulus had no observable effect on reflex amplitude in either the A-A or the C-A condition. The main effect of $\mathrm{S} 2$ frequency was not significant $[F(4,28)<1]$.

Reflex latency. Mean reflex latencies from the two conditions at the five $S 2$ frequencies and for the single acoustic and cutaneous stimuli are shown in Figure 4. The frequency of the second stimulus had no systematic effect on reflex latency in either stimulus pair condition. The main effect of S2 frequency was not significant $[F(4,28)<1]$.

The main effect of stimulus condition was significant $[F(1,7)=6.26, p<.05]$, but the interaction of stimulus condition and $S 2$ frequency was not $[F(4,28)=1.03$, $p<.05]$. The reflex latency averaged across the five $\mathrm{S} 2$ frequencies in the A-A condition was $48 \mathrm{msec}$, which was the same as the single-stimulus control latency. The corresponding latencies from the $\mathrm{C}-\mathrm{A}$ condition were 51 and $53 \mathrm{msec}$. This 2 -msec difference was smaller than that

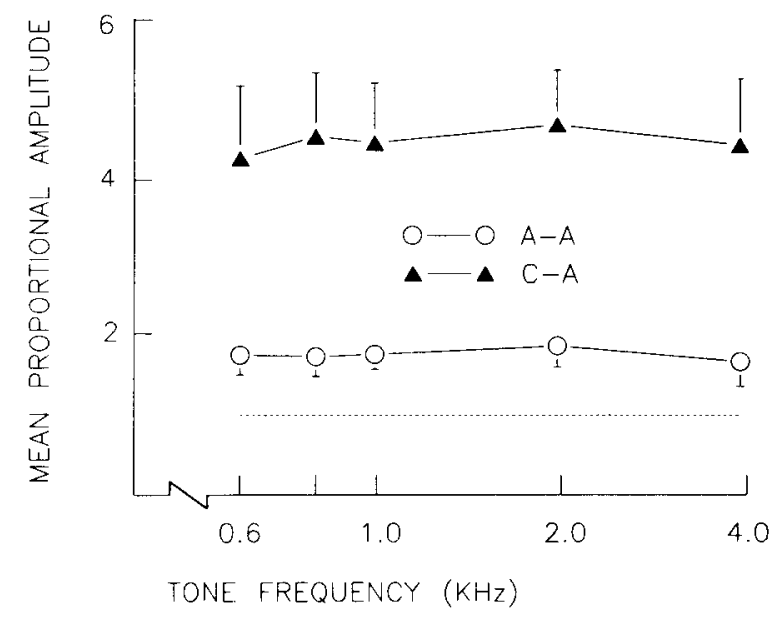

Figure 3. Geometric means of the proportional reflex amplitudes for each of the five $\mathbf{S 2}$ tone frequencies in Experiment 2. The dotted line shows a proportional amplitude of 1.0. The vertical bars show one standard error of the mean.

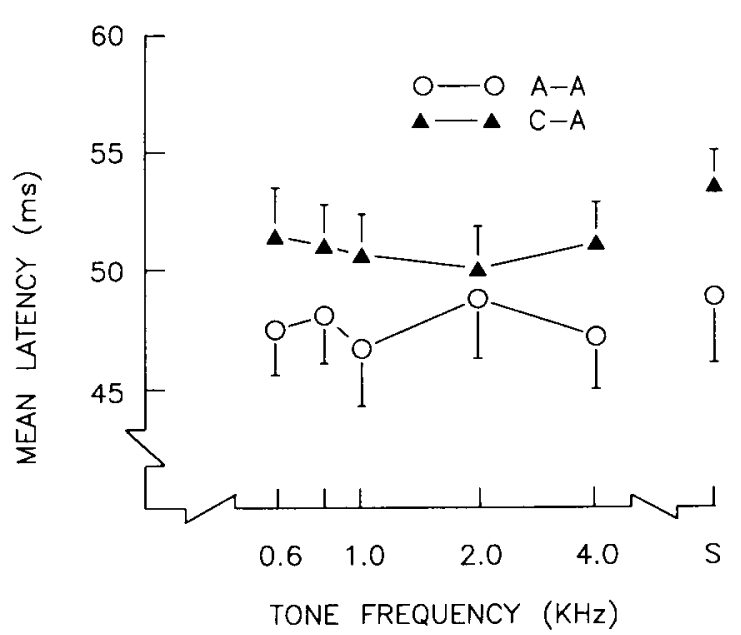

Figure 4. Mean reflex latencies (measured in milliseconds from the first stimulus) for each of the five $\mathbf{S 2}$ tone frequencies in Experiment 2. Single-stimulus latencies (S) for the acoustic stimuli (open circle) and cutaneous stimuli (filled triangle) are shown on the right of the figure. The vertical bars show one standard error of the mean.

seen in Experiment 1 (where it was $3 \mathrm{msec}$ ) and was shown by one-tailed Dunnett's tests to be significant only for three of the five $S 2$ frequencies.

Loudness estimation. Loudness was an inverted Ushaped function of stimulus frequency. Average magnitude estimations of the four $\mathbf{S} 2$ frequencies as percentages of the loudness of the $4-\mathrm{kHz} \mathrm{S} 1$ were as follows: $0.6 \mathrm{kHz}=83 \%(S E=3), 0.8 \mathrm{kHz}=115 \%(S E=3)$, $1.0 \mathrm{kHz}=117 \%(S E=2)$, and $2.0 \mathrm{kHz}=91 \%$ $(S E=2)$. The effect of stimulus frequency on loudness was significant $[F(3,28)=4.73, p<.05]$.

\section{Discussion}

The results of Experiment 2 replicate the findings of Experiment 1 in two ways. First, reflex amplitude is augmented crossmodally as well as intramodally, and second, it is greater with heterogeneous stimulus pairs. Latency facilitation was also present, although less pronounced than in Experiment 1.

The blink reflexes elicited by the first stimulus in both stimulus-pair conditions were unaffected by the frequency of the second stimulus, which was varied over a sufficiently wide range to release any masking that might have occurred between tones of the same frequency (Green \& Wier, 1984). Although variations in S2 frequency resulted in loudness changes, these would not have compensated for the progressive release from masking that would be expected with the $S 2$ frequency variations. Masking is therefore unlikely to be responsible for the smaller amplitude enhancement observed in homogeneous than in heterogeneous stimulus pairs.

\section{GENERAL DISCUSSION}

The present results and those of Blumenthal and W. K. Berg (1986) show the critical duration of integration of the effects of two successive acoustic blink-eliciting 
stimuli on reflex amplitude to be less than $50 \mathrm{msec}$. The critical duration for latency is shorter, probably less than $20 \mathrm{msec}$. These values are much shorter than psychophysical estimates of critical durations for temporal integration in the auditory system. Recent reviews of the psychoacoustical literature (Scharf \& Buus, 1986; Scharf \& Houtsma, 1986) show lowered detection thresholds for paired tone bursts separated by intervals up to about $100 \mathrm{msec}$ and, at suprathreshold levels, loudness summation of tone bursts over silent intervals of about $200 \mathrm{msec}$. The disparate time courses of temporal integration of reflexogenic stimuli shown in reflex expression and the temporal integration shown in psychophysical judgments suggest that the two methods reflect different aspects of stimulus processing. Temporal integration of reflexogenic stimuli may result from summation of activity associated more closely with motor outflow than with perceptual awareness.

The general correspondence of the upper limit of the temporal integration window and the reflex latency evident in this study and in that of Blumenthal and W. K. Berg (1986) is also evident in rat whole-body acoustic startle, in which the upper limit of temporal integration has been reported as $8 \mathrm{msec}$ (Marsh et al., 1973) and the latency as varying from 6 to $12 \mathrm{msec}$ (Ison, McAdam, \& Hammond, 1973). It appears that following a reflexeliciting stimulus, a second reflex-eliciting stimulus presented before response initiation enhances the reflex elicited by the first member of the pair. This observation, together with the finding of integration of stimuli from different modalities, supports the suggestion that reflex enhancement with pairs of successive reflexogenic stimuli is a result of integration of activity associated closely with reflex elicitation.

Measures of reflex activity can, however, give objective measures of sensory processing that parallel those from conventional psychophysical procedures. Reflex amplitude and latency are altered systematically by nonreflexogenic stimuli that precede reflex elicitation (Hoffman \& Ison, 1980). This phenomenon can be used to study processing of the leading reflex-modifying stimuli. Dykman and Ison (1979), for example, have shown that the reflex-modifying effects of pairs of leading stimuli summate over intervals as long as $\mathbf{2 0 0} \mathrm{msec}$, an interval longer than that found for integration of pairs of reflexogenic stimuli and consistent with the psychophysical estimates of temporal integration.

Motor outflow for the cutaneous blink reflex is organized in the lower brainstem reticular formation (Ongerboer de Visser \& Kuypers, 1978). Although the pathway for the acoustic blink reflex has not been described completely, it is likely to involve the same brainstem reticular structures; this is the case at least for the acoustic startle reflex in cats (Davis, Gendelman, Tischler, \& Gendelman, 1982). The reticular response-organizing system may be the site of temporal integration of successive reflexogenic stimuli. Electrophysiological studies have shown that many cells throughout the reticular formation are polysensory (e.g., Amassian \& Devito, 1954; Bell, Sierra, Buendia, \& Segundo, 1964), a property that results from convergence of multiple afferents onto single reticular cells (Zimmerman, 1981). This property makes these cells suitable for crossmodal as well as intramodal integration. The greater amplitude augmentation found with heterogeneous stimulus pairs might also result from interaction of the stimuli within the response-organizing system in the lower brainstem reticular formation. Fox and Wolstencroft (1976) reported that medullary reticular cells showed greater responsitivity to the second member of a stimulus pair when each member was applied to separate, rather than the same, afferent branches. They proposed this as a cellular mechanism for novelty detection by reticular cells. It may also be a mechanism for the greater amplitude augmentation seen with heterogeneous stimulus pairs: after firing to a stimulus, neurons of the response-organizing system may be more responsive to a second stimulus in a different sensory modality than to a second stimulus in the same sensory modality.

The mechanism of integration of pairs of reflexogenic stimuli may be simple summation of activity arriving at the reticular neurons involved in reflex expression. Both amplitude and latency would be enhanced when activity evoked by the second stimulus arrives at these cells before the peak of activity evoked by the first stimulus. In this case, the summated activity would be greater and would have a steeper onset than that evoked by a single stimulus. The greater activity would result in more cells firing and increase reflex amplitude; assuming that some threshold level of activity must be reached before a measurable response is triggered, the steeper onset of activity would shorten reflex latency. When the activity evoked by the second stimulus arrives after the peak of activity evoked by the first stimulus, but before the activity decays, the summated activity would be greater than that evoked by a single stimulus (and reflex amplitude would be augmented), but the onset rate of the activity would be unaffected (and hence reflex latency would be unchanged). This simple view of integration accounts for the different temporal limits on reflex amplitude and latency without supposing that these two response characteristics are determined by different mechanisms.

\section{REFERENCES}

Amassian, V. E., Devito, R. V. (1954). Unit activity in reticular formation and nearby structures. Joumal of Neurophysiology, 17, 575-603.

Bell, C., Sierra, G., Buendia, N., \&egundo, J. P. (1964). Sensory properties of neurons in the mesencephalic reticular formation. Journal of Neurophysiology, 27, 961-987.

BERG, K. M. (1973). Elicitation of acoustic startle in the human. Dissertation Abstracts International, 34, 5217B-5218B.

Blumenthal, T. D., Berg, W. K. (1986). The startle response as 
an indicator of temporal summation. Perception \& Psychophysics, 40, 62-68.

D'Aмато, M. R. (1979). Experimental psychology: Methodology. psychophysics, and learning. New Delhi: Tata McGraw-Hill.

Davis, M., Gendelman, D. S., Tischler, M. D., Gendelman, P. M. (1982). A primary acoustic startle circuit: Lesion and stimulation studies. Journal of Neuroscience, 2, 791-805.

DYKMAN, B. M., \& IsON, J. R. (1979). Temporal integration of acoustic stimulation obtained in reflex inhibition in rats and humans. Journal of Comparative \& Physiological Psychology, 93, 939-945.

Fox, J. E., \& Wolstencroft, J. H. (1976). The reduced responsiveness of neurons in nucleus reticularis gigantocellularis following their excitation by peripheral nerve stimulation. Journal of Physiology, 258, 687-704.

GersuNI, G. V. (1971). Temporal organization of the auditory function. In G. V. Gersuni (Ed.), Sensory processes at the neuronal and behavioral levels (pp. 85-114). New York: Academic Press.

GREEN, D. M., \& WER, C. C. (1984). Auditory perception. In I. DarianSmith (Ed.), Handbook of physiology: Sec. I. The nervous system: Vol. III, Pt. 2. Sensory processes (pp. 557-594). Bethesda, MD: American Physiological Society.

HofFMan, H. S., \& IsON, J. R. (1980). Reflex modification in the domain of startle: I. Some empirical findings and their implications for how the nervous system processes sensory input. Psychological Review, 87, 175-189.

Ison, J. R., McAdam, D. W., \& Hammond, G. R. (1973). Latency and amplitude changes in the acoustic startle reflex of the rat produced by variation in auditory prestimulation. Physiology \& Behavior, 10, 1035-1039.

Marsh, R., Hoffman, H. S., \& Stitt, C. L. (1973). Temporal integration in the acoustic startle reflex of the rat. Journal of Comparative \& Physiological Psychology, 82, 507-511.

ONgerboer DE VisSer, B. W., \& KUYPERS, H. G. J. M. (1978). Late blink reflex changes in lateral medullary lesions: An electrophysiological and neuro-anatomical study of Wallenberg's syndrome. Brain, 101, 285-294.

Penders, C. A., Delwaide, P. J. (1973). Physiologic approach to the human blink reflex. In J. E. Desmedt (Ed.), New developments in electromyography and clinical neurophysiology: Vol. 3. Human reflexes, pathophysiology of motor systems, methodology of human reflexes. (pp. 649-657). Basel: Karger.

Radionova, E. A. (1971). Two types of neurons in the cat cochlear nuclei and their role in audition. In G. V. Gersuni (Ed.), Sensory processes at the neuronal and behavioral levels ( $\mathrm{pp}$. 135-155). New York: Academic Press.

SCharf, B., \& BuUs, S. (1986). Audition I. In K. R. Boff, L. Kaufman, \& J. P. Thomas (Eds.), Handbook of perception and human performance: Vol. I. Sensory processes and perception (pp. 14.114.71). New York: Wiley.

Scharf, B., Houtsma, A. J. M. (1986). Audition II. In K. R. Boff, L. Kaufman, \& J. P. Thomas (Eds.), Handbook of perception and human performance: Vol. 1. Sensory processes and perception (pp. 15.1-15.60). New York: Wiley.

Shahani, B. T., \& Young, R. R. (1973). Blink reflexes in orbicularis oculi. In J. E. Desmedt (Ed.), New developments in electromyography and clinical neurophysiology: Vol. 3. Human reflexes, pathophysiology of motor systems, methodology of human reflexes (pp. 641-648). Basel: Karger.

ZimmermanN, M. (1981). Neurophysiology of sensory systems. In R. R. Schmidt (Ed.), Fundamentals of sensory physiology (2nd ed., pp. 3180) (M. A. Biederman-Thorson, Trans.). Heidelberg: SpringerVerlag.

(Manuscript received February 16, 1988; revision accepted for publication September 27, 1988.) 